\title{
On the enumeration of Boolean functions with distinguished variables
}

\author{
Josep Freixas
}

Received: date / Accepted: date

\begin{abstract}
Boolean functions have a fundamental role in neural networks and machine learning. Enumerating these functions and significant subclasses is a highly complex problem. Therefore, it is of interest to study subclasses that escape this limitation and can be enumerated by means of sequences depending on the number of variables.

In this article we obtain seven new formulas corresponding to enumerations of some subclasses of Boolean functions. The versatility of these functions does the problem interesting to several different fields as game theory, hypergraphs, reliability, cryptography or logic gates.
\end{abstract}

Keywords Boolean functions - 2-monotonic Boolean functions - Simple games · Complete games $\cdot$ Enumeration of Boolean Functions $\cdot$ Dedekind's problem

Mathematics Subject Classification (2010) 06E30 - 90B25 - 91A12 - 91A80 • 91B12 $\cdot 94 \mathrm{C} 10$

\section{Introduction}

Since its origins, mathematics has been interested in classifying and listing the set of solutions to a given problem. As quoted in [17] 'to enumerate a set of objects satisfying some set of properties means to explicitly produce a listing of all such objects'. Enumerating special types of Boolean functions or simple games is useful for the design of circuits and real-world voting systems that fulfil some desirable properties. This paper concerns enumerations of these structures.

J. Freixas

Av. Bases de Manresa, 61-73, 08242 Manresa, Spain. Universitat Politècnica de Catalunya.

Tel.: +34-938777246

E-mail: josep.freixas@upc.edu 
A Boolean function has as input $n$ Boolean variables (that is, values that can be either false or true) and produces as output another Boolean variable. It is monotonic if, for every combination of inputs, switching one of the inputs from false to true can only cause the output to switch from false to true and not from true to false. More precisely, a monotonic Boolean function of $n$ variables (or, for short, a function) is a mapping $f:\{0,1\}^{n} \rightarrow\{0,1\}$ such that: $x \leq y$ implies $f(x) \leq f(y)$. The Dedekind's problem is given by the sequence $M(n)$ and is the number of monotonic Boolean functions of $n$ variables, or the number of antichains of subsets of an $n$ set, or the number of elements in a free distributive lattice on $n$ generators, or the number of Sperner families. Recall that an antichain of sets (also known as a Sperner family) is a family of sets, none of which is contained in any other set. The values of the sequence $M(n)$ for the first eight integers are known: 2, 3, 6, 20, 168, 7581, 7828354, 2414682040998, 56130437228687557907788 (see sequence A000372 in the On-Line Encyclopedia of Integer Sequences, [34]).

The (inequivalent) Dedekind's number $S(n)$ is the number of different monotonic Boolean functions on $n$ variables that do not differ in the name of the variables. If two Boolean functions are only differentiated in the labels, they are said to be equivalent. Thus, $S(n)$ counts the number of inequivalent monotonic Boolean functions of $n$ or fewer variables. The values of this sequence for the first eight integers are also known: 2, 3, 5, 10, 30, 210, 16353, 490013148 (see sequence A003182 in the OnLine Encyclopedia of Integer Sequences, [34]). This paper concerns enumerations of subclasses of inequivalent monotonic Boolean functions.

Two subclasses that excel within inequivalent monotonic Boolean functions are: the threshold functions and the 2-monotonic (or regular) functions, a superclass of threshold functions. A function $f$ is called 2-monotonic or regular if it satisfies the following condition at every $x$ : if $i<j, x_{i}=1, x_{j}=0$ and $f(x)=0$, then $f(x+$ $\left.e_{j}-e_{i}\right)=0$, where $e_{k}$ denotes the $k$-th unit vector of appropriate dimension. 2monotonicity and related concepts have been studied under various names in such areas of applied mathematics as threshold logic ([18, 28]), game theory ( [3,7],42]) or graph and hypergraph theory ( [5, 32] ). The interest in 2-monotonic functions usually stems from their close relationship with threshold functions, a Boolean function $f(x)$ is called threshold if there exist $n+1$ integers $w_{1} \geq w_{2} \geq \cdots \geq w_{n} \geq 0$ (weights) and $q>0$ (quota or threshold) such that: $f(x)=1$ if and only if $\sum_{i=1}^{n} w_{i} \cdot x_{i} \geq q$.

The number of threshold functions and 2-monotonic functions are known up to $n=9$ variables, see respectively [13] and [22,24]. Identification of 2-monotonic Boolean functions can be done in polynomial time [1,2] and computational studies of separation algorithms for clique inequalities can serve for determining threshold functions [27].

Recent research in capacitive threshold logic, strongly studied in the sixties, see e.g., [18], has revived interest in this area, and it has reintroduced some of the problems that have yet to be solved. One of the main issues of threshold logic is the application of neural networks to the problem of realizing Boolean functions (that is, to decide if a given Boolean function is threshold). The linear separability problem has 
been dealt with, among others, in [10, 12, 30, 33, 35, 40, 42]. Neural networks are usually designed to have the ability to learn and generalize. Neural networks have been used with computers during as early as the 1950s. Through the years many different neural network architectures have been presented. One of the pioneering contributions was the perceptron, which is the simplest form of a neural network used for the classification of linearly separable patterns (patterns that are located on the opposite sides of a hyperplane). Basically, it consists of a single neuron with adjustable weights and a threshold, that is, a threshold function. The algorithm used to adjust the free parameters of this network emerged as a Rosenblatt learning procedure for his model of the brain, the perceptron. The convergence proof of such algorithm is known as the Perceptron Convergence Theorem.

Another criterion for choosing significant subclasses of inequivalent monotonic Boolean functions consists in considering the number of types of equivalent variables. Two variables that play the same role in the function are considered equivalent, then the set of variables decomposes into equivalence classes. Functions with a moderate number of equivalence classes are very useful in applications. Inequivalent functions with two equivalence classes are called bipartite. Similarly, tripartite and quadripartite inequivalent Boolean functions are considered. Quite curiously, bipartite 2-monotonic Boolean functions are enumerated for all $n$ and follow a variant of a Fibonacci sequence (see [13] and equation (1) in this paper) and therefore grow exponentially on the number of variables $n$. Bipartite inequivalent Boolean functions were enumerated recently in [15] after obtaining the enumeration of bipartite functions non-being 2-monotonic. The purpose of this paper is to enumerate some tripartite and quadripartite inequivalent Boolean functions with dominant and / or dominated variables.

Boolean functions are very versatile structures that arise in many contexts and lend themselves to more diverse interpretations. Monotonic Boolean functions are equivalent to monotonic hypergraphs that can also be thought as simple games, coherent structures (see e.g., [31]), logic gates or reliability systems (see e.g. [21], and [14] where basic analogies between reliability and game theory were listed), access structure in a secret sharing (see e.g., [16 36-38]), etc. Each of these fields have developed different theories motivated by their discipline challenges, so that a fruitful cooperation has taken place allowing for a great development in this area of research.

Simple games are at the core of voting systems, in them a single alternative, such as a bill or an amendment, is pitted against the status quo, and voters can vote for or against the bill. Due to its importance, naturalness and significance of its applications (see e.g., [l $\left.\begin{array}{lllllllllllll}6 & 8 & 9 & 20 & 23 & 25 & 26 & 39 & 41 & 43\end{array}\right]$, we have adopted in this paper the language of simple games. We also point out that a simple game can be seen as a particular case of a cooperative game, see e.g., [29], whenever the image set of the characteristic function $v: 2 N \rightarrow \mathbb{R}$ is binary, usually described by $\{0,1\}$, in which the value 0 is interpreted as a losing result and the value 1 as a winning result, so that any coalition $S$ with $v(S)=0$ is losing and any coalition with $v(S)=1$ is winning. Nevertheless, the theory of simple games has been mostly developed independently of cooperative games since the outputs 'losing' and 'winning' are qualitative rather than quantitative as occurs for cooperative games. 
We consider that this paper is relevant for the readers of Soft Computing because the most relevant problem in Boolean functions is the separability problem, that consists in determining whether a given function is threshold. As 2-monotonicity is a necessary, but not sufficient condition for the function to be threshold, it is of great interest to enumerate all 2-monotonic functions and then check one-by-one which of them are threshold since this checking can be done in polynomial time, cf. [4]. Nevertheless, it is a very complex problem listing 2-monotonic functions. Developments of techniques in Soft Computing may be helpful in future developments.

The paper is organized as follows. Section 2 sets out the main terminology and notions that will be used in the article. Section 3 collects the known results on enumerations for bipartite, tripartite and quadripartite simple games. Sections 4, 5 and 6 are devoted to find new enumerations of tripartite simple games with either vetoes or nulls. Sections 7, 8 and 9 are devoted to find new enumerations of quadripartite simple games with vetoes and nulls. Section 10 concludes the paper by summarizing the main findings and indicating some open problems to encourage future research.

\section{Background on simple games}

Definition 1 A simple game is a pair $(N, W)$ in which $N=\{1,2, \ldots, n\}$ and $W$ is a collection of subsets of $N$ that satisfies: $N \in W, \emptyset \notin W$ and, the monotonicity property, if $S \in W$ and $S \subseteq T \subseteq N$ then $T \in W$.

A (monotonic) simple game corresponds to a (monotonic) Boolean function in the field of Boolean algebra.

The set $N$ is called the grand coalition. Members of $N$ are called players or voters, and subsets of $N$ are called coalitions, the coalitions that belong to $W$ are called winning coalitions, the subfamily of minimal winning coalitions is $W^{m}=$ $\{S \in W: \forall T \subset S \Rightarrow T \notin W\}$. The minimal winning coalitions form an antichain of subsets that allows to generate the simple game. By $|S|$ we mean the cardinality of a coalition $S$ and use $|S|=s$ whenever there is no confusion.

Definition 2 Two simple games $(N, W)$ and $\left(N^{\prime}, W^{\prime}\right)$ are isomorphic if there exists a one-to-one correspondence $f: N \rightarrow N^{\prime}$ such that $S \in W$ if and only if $f(S) \in$ $W^{\prime} ; f$ is called and isomorphism of simple games.

Two isomorphic simple games only differ in the labels, so from now on we will only consider simple games up to isomorphisms. (Monotonic) simple games up to isomorphisms correspond to inequivalent (monotonic) Boolean functions. Two distinguished types of voters frequently appear in simple games.

Definition 3 A voter $i$ has veto whenever $i \in S$ for all $S \in W$. A voter $i$ is null whenever $i \notin S$ for all $S \in W^{m}$. 
In any democratic voting system represented by a simple game, the veto players (if any) are the most powerful ones in the system. Oppositely, null voters (if any) do not exert any influence in the game. In Boolean algebra the null voters correspond to irrelevant variables and the veto players to essential variables.

One common idea of the most voting systems used in practice is the concept of influence, i.e., that a particular voting system may give one voter more influence than another. The so-called 'desirability' relation, precisely stated in Definition 4 , on the set of voters is a way to make influence precise. Isbell already used it in [19].

Definition 4 Let $(N, W)$ be a simple game. Player $i$ is at least as desirable as $j$ ( $i \succsim j$, in short) in $(N, W)$ if: $S \cup\{j\} \in W \Rightarrow S \cup\{i\} \in W$, for all $S \subseteq N \backslash\{i, j\}$. Players $i$ and $j$ are equally desirable $(i \approx j$, in short) in $(N, W)$ if: $i \succsim j$ and $j \succsim i$. Player $i$ is strictly more desirable than player $j(i \succ j$, in short) in $(N, W)$ if: $i \succsim j$ and $i \not j$.

Note that the desirability relation is a preordering, a reflexive and transitive relation, on the set of players $N$ and that each subset $N_{i} \subseteq N$ fomed by equally desirable players is an equivalence class. We refer to $N_{i}$ as an equally desirable class. We also speak of the number of classes or types, $t$, of voters meaning the number of equivalence classes. This is a fundamental parameter in our study. The veto players (if any) form the strongest equivalence class, $N_{1}$, whereas null players (if any) form the weakest equivalence class, $N_{t}$.

Definition 5 A simple game $(N, W)$ is complete or linear if the desirability relation is a complete preordering.

Complete simple games correspond to 2-monotonic Boolean functions in Boolean algebra. Let $t$ be the number of types of a complete game, we can always assume $N_{1}>N_{2}>\cdots>N_{t}$ understanding that $N_{k}>N_{k+1}$ if and only if $i \succ j$ for all $i \in N_{k}$ and $j \in N_{k+1}$.

Definition 6 Let $(N, W)$ be a simple game with $t$ equally desirable classes. If $t=2$ the game is called bipartite, if $t=3$ it is called tripartite and if $t=4$ it is called quadripartite.

In this paper we are concerned with tripartite simple games having either voters with right to veto (vetoes, for short) or null voters (nulls, for short) and with quadripartite simple games having vetoes and nulls. As we shall see in Proposition 11, all the enumerations of this paper can be done assuming the presence of veto players in the game. Thus, for the tripartite case we will have: $N_{1}>N_{2}>N_{3}$ if the game is complete and $N_{1}>N_{i}$ for $i=2,3$ if the game is not complete because $N_{1}$ is formed by vetoes. For quadripartite games with vetoes (voters in $N_{1}$ ) and nulls (voters in $N_{4}$ ) we will have $N_{1}>N_{2}>N_{3}>N_{4}$ if the game is complete and $N_{1}>N_{i}$ for $i=2,3,4$ and $N_{i}>N_{4}$ for $i=2,3$ if the game is not complete, but neither $N_{2}>N_{3}$ nor $N_{3}>N_{2}$ are met. 
Example 1 Let $N$ be a Parliament formed by the president and two chambers, for example the House and the Senate. To pass a proposal it is required the approval of the president and the approval of an absolute majority of the members in each chamber. Assume the sizes of the chambers are 9 and 15, thus, $|N|=25$. Then, the approval is achieved if the president votes in favor, at least 5 members of the House vote in favor and at least 8 members of the Senate vote in favor of the proposal submitted at hand. Let $N_{1}=\{1\}, N_{2}=\{2, \ldots, 10\}$ and $N_{3}=\{11, \ldots, 25\}$, where 1 represents the president, the elements in $N_{2}$ are those in the House, and the elements in $N_{3}$ are those in the Senate. Formally,

$$
W^{m}=\left\{S \subseteq N: 1 \in S,\left|S \cap N_{2}\right|=5 \text {, and }\left|S \cap N_{3}\right|=8\right\} .
$$

Note that: the president has veto because $1 \in S$ for all $S \in W$ and $1 \succ i$ for all $i \in N \backslash\{1\}$, but neither $i \succsim j$ nor $j \succsim i$ for all $i \in N_{2}$ and $j \in N_{3}$. Thus, the game is a tripartite non-complete game with a veto player.

A simple game can be presented in a more convenient way. Given the equivalence classes of the simple game $N_{1}, \ldots, N_{t}$ such that $N_{i+1}>N_{i}$ does not hold for every $i=1, \ldots, t-1$, the vector $\bar{n}=\left(n_{1}, n_{2}, \ldots, n_{t}\right)$ represents the grand coalition and any vector $\bar{s}=\left(s_{1}, s_{2}, \ldots, s_{t}\right)$ with $0 \leq s_{i} \leq n_{i}$ represents the set of coalitions $\left\{S:\left|S \cap N_{i}\right|=s_{i}\right.$, for all $\left.i=1, \ldots, t\right\}$ identified with $\bar{s}$. Coalitions represented by the model $\bar{s}$ are either all winning or all are not. Thus, we write either $\bar{s} \in \bar{W}$ or $\bar{s} \notin \bar{W}$, where $\bar{W}=\{\bar{s} \in \Lambda(\bar{n}): S \in W\}$ and $\Lambda(\bar{n})$ is the set of models of coalitions, i.e., $\left\{\bar{m} \in(\mathbb{N} \cup\{0\})^{t}: \overline{0} \leq \bar{m} \leq \bar{n}\right\}$. The elements in $\bar{s} \in \bar{W}$, which are minimal componentwise, represent minimal winning coalitions and write $\bar{s} \in \overline{W^{m}}$ for them, where $\overline{W^{m}}=\left\{\bar{s} \in \Lambda(\bar{n}): S \in W^{m}\right\}$.

Let $\bar{m} \in \Lambda(\bar{n})$ and $\bar{p} \in \Lambda(\bar{n})$. By $\bar{m} \leq \bar{p}$ we mean $m_{i} \leq p_{i}$ for all $i=1, \ldots, t$, and by $\bar{m}<\bar{p}$ we mean $\bar{m} \leq \bar{p}$ and $\bar{m} \neq \bar{p}$. By $\overline{0}$ we mean the $t$-dimensional vector formed by zeros. The next conditions meet the requirements of monotonic simple games:

(i) $\bar{n} \in \bar{W}$

(ii) $\overline{0} \notin \bar{W}$, and

(iii) $\bar{m} \leq \bar{p}$ with $\bar{m} \in \bar{W}$ implies $\bar{p} \in \bar{W}$.

See [3] for further details on this presentation of simple games. If we know that $N_{i}>N_{i+1}$, then the next condition is also fulfilled:

(iv) Let $\bar{m}=\left(m_{1}, \ldots, m_{i-1}, m_{i}, m_{i+1}, \ldots, m_{t}\right) \in \bar{W}$ with $m_{i}<n_{i}$ and $m_{i+1}>$ 0 , then $\left(m_{1}, \ldots, m_{i-1}, m_{i}+1, m_{i+1}-1, \ldots, m_{t}\right) \in \bar{W}$.

Note that condition $(i v)$ captures the idea that if a player is replaced in a winning coalition by another player being more desirable than her, then the new coalition becomes also winning. Observe that in this argument the condition $N_{i}>N_{i+1}$ is essential.

Another fundamental parameter in our study for each game is $r$, the number of representatives of minimal winning coalitions of the simple game, that is the cardinality of $\overline{W^{m}}$. 
The following nomenclature is used in the rest of the paper. Each description depends on either $(n, r)$ or simply $n$. By $(n, r)$ we refer to $n$ voters and $r$ representatives of minimal winning coalitions.

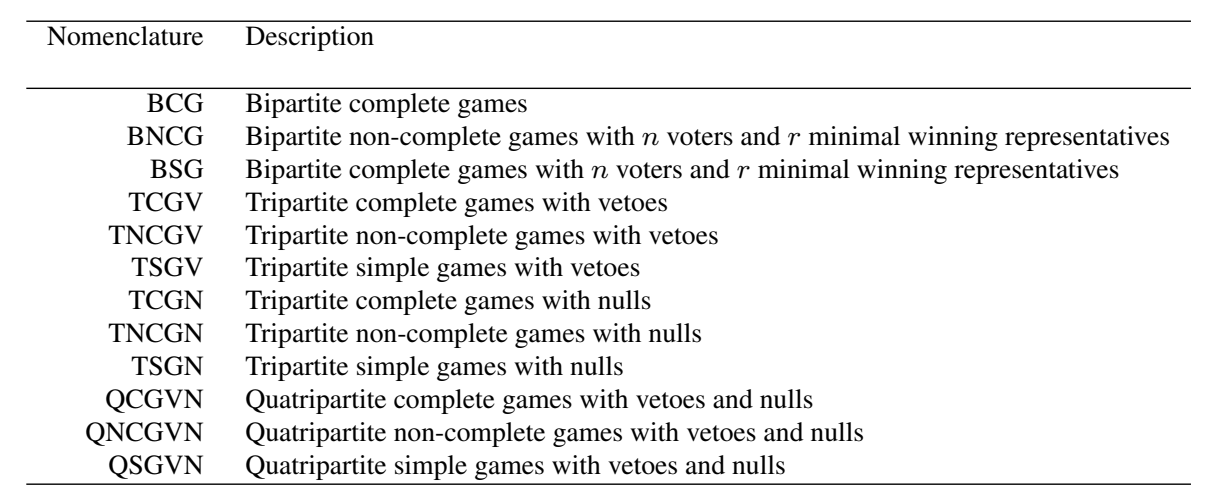

Table 1 The classes of games we deal with in this paper.

Thus, by $\operatorname{BCG}(n, r)$ we mean the class of all bipartite complete games with $n$ voters and $r$ representatives of minimal winning coalitions, up to isomorphisms, and by $B C G(n, r)$ its cardinality. $\operatorname{By} \operatorname{BCG}(n)$ we mean the class of all bipartite complete games with $n$ voters, up to isomorphisms, and by $B C G(n)$ its cardinality. The rest of notations in Table 1 are analogous.

Example 2 (Example 1 1 revisited) As noticed, the game belongs to TNCGV for $n=$ 25 and $r=1$ since the game has 25 players and there is only one representative of minimal winning coalitions, which is $(1,5,8)$. The winning representatives are the vectors in the set:

$$
\{(1, h, s) \in \Lambda(1,9,15): 5 \leq h \leq 9 \text { and } 8 \leq s \leq 15\} .
$$

Note that there are $\left(\begin{array}{l}1 \\ 1\end{array}\right) \cdot\left(\begin{array}{l}9 \\ 5\end{array}\right) \cdot\left(\begin{array}{c}15 \\ 8\end{array}\right)=810810$ minimal winning coalitions with representative $(1,5,8)$. This simple example illustrates the convenience of using the succinct representation of the game by the vector $\bar{n}$ and the set $\overline{W^{m}}$ instead of the standard presentation given by the set of voters $N$ and the set of minimal winning coalitions $W^{m}$.

It obviously holds the following property:

Proposition 1 For all $n$ and $r$ we have:

a. $T C G V(n, r)=T C G N(n, r)$

b. $T N C G V(n, r)=T N C G N(n, r)$

Proof. Let $(N, W) \in \operatorname{TCGV}(n, r)$ and without null voters, let $\left(N, W^{\prime}\right)$ be obtained from $(N, W)$ defined as

$$
S \in W^{\prime} \text { if and only if } S \cup N_{1} \in W
$$


so that the vetoes in $N_{1}$ for $(N, W)$ have been converted into nulls in $\left(N, W^{\prime}\right)$. The 'if and only if' clause guarantees the one-to-one correspondence. The reasoning done does not depend on $n$ or $r$. The second part is mutatis mutandis the same.

Corollary 1 For all $n$ and $r$ we have:

a. $T S G V(n, r)=T S G N(n, r)$,

b. $T C G V(n)=T C G N(n)$,

c. $T N C G V(n)=T N C G N(n)$, and

d. $T S G V(n)=T S G N(n)$.

Hence, by Proposition 1 and Corollary 1, the enumerations for tripartite games with vetoes for either complete or non-complete games coincide with the respective enumerations for tripartite games with nulls for complete and non-complete games.

\section{Known enumerations for simple games with less than 5 equivalence classes}

If only anonymous or symmetric (i.e., any pair of voters are equally desirable) voters are considered for simple games with $n$ voters we have $t=1$ and $U S G(n)=$ $U C G(n)=n$, here $U S G(n)$ denotes the number of unipartite simple games and $U C G(n)$ the number of unipartite complete games.

The number of bipartite complete games with $n$ voters, $B C G(n)$, were enumerated in [13] and later on in [24], giving a proof based on generating functions:

$$
B C G(n)=F(n+6)-\left(n^{2}+4 n+8\right),
$$

where $F(n)$ are the Fibonacci numbers which constitute a well-known sequence of integer numbers defined by the following recurrence relation: $F(0)=0, F(1)=1$, and $F(n)=F(n-1)+F(n-2)$ for all $n>1$ from which the formula

$$
F(n)=\frac{1}{\sqrt{5}}\left[\left(\frac{1+\sqrt{5}}{2}\right)^{n}-\left(\frac{1-\sqrt{5}}{2}\right)^{n}\right], \quad n \geq 0
$$

is deduced.

The number of tripartite complete games with vetoes and $n$ voters, $\operatorname{TCGV}(n)$, (and the number of tripartite complete games with nulls and $n$ voters, TCGN $(n)$ ), were enumerated in [11]:

$$
\operatorname{TCGV}(n)=\operatorname{TCGN}(n)=F(n+7)-\frac{1}{2}\left(n^{3}+2 n^{2}+13 n+26\right),
$$

whenever $n \geq 4$.

The number of quadripartite complete games with vetoes and nulls with $n$ voters, $\operatorname{QCGVN}(n)$, were also enumerated in [11]:

$$
\operatorname{QCGVN}(n)=\operatorname{CGVN}(n, 4)=F(n+8)-\frac{1}{6}\left(n^{4}-2 n^{3}+26 n^{2}+47 n+132\right)
$$


whenever $n \geq 5$.

Note that $B C G(n), \operatorname{TCGV}(n)$, and $Q C G V N(n)$ belong to $\Theta\left(\left(\frac{1+\sqrt{5}}{2}\right)^{n}\right)$, which means that the limits:

$$
\lim _{n \rightarrow \infty} \frac{B C G(n)}{((1+\sqrt{5}) / 2)^{n}}, \quad \lim _{n \rightarrow \infty} \frac{\operatorname{TCGV}(n)}{((1+\sqrt{5}) / 2)^{n}}, \quad \lim _{n \rightarrow \infty} \frac{\operatorname{QCGVN}(n)}{((1+\sqrt{5}) / 2)^{n}}
$$

are real numbers. This is because the sequences in the numerators are functions of $n$ which are the sums of two exponentials with different basis and a polynomial. As $n$ tends to infinity, one may disregard the exponential term with lower basis in absolute value and the polynomial. In particular, the three respective limits in (4) are the positive numbers: $9+4 \sqrt{5},(1 / 2)(29+13 \sqrt{5})$, and $(1 / 2)(47+21 \sqrt{5})$.

The sequence $B C G(n)$ appears in [34] with code A163250, but also in A053808, which suggests another equivalent way to interpret the numbers in the first column of Table 3 .

The combinations of $n$ and $r$ that give rise to non-complete games are determined by the following result in [15], which will be used from Section 4 to Section 9. The following trivial lemma provides an upper bound for $r$ as a function of $n$.

Lemma $1 B N C G(n, r) \neq 0$ if and only if $n>3$ and $r \leq\left\lfloor\frac{n}{2}\right\rfloor$.

From Lemma 1 the following result was immediately deduced.

\section{Corollary 2}

$$
B N C G(n)= \begin{cases}0 & \text { if } n \leq 3 \\ \sum_{r=1}^{\lfloor n / 2\rfloor} B N C G(n, r) & \text { if } n>3\end{cases}
$$

The number of bipartite non-complete games with $n$ voters, $B N C G(n)$, was recently studied in [15]. A parameterization for these games allows to generate all games of this type for small values of $n$ and $r$. Table 2 shows the positive numbers $B N C G(n, r)$ for all pairs $(n, r)$ with $n<14$. The first column indicates the number of players, $n$, and the first row the number of representatives of minimal winning coalitions, $r$. The numbers in Table 2 together with those for complete games in (1) allow to determine the number of bipartite simple games $B S G(n)$ for small values of $n$, see Table 3 . 


\begin{tabular}{crrrrrr}
\hline $\mathrm{n} \downarrow / \mathrm{r} \rightarrow$ & 1 & 2 & 3 & 4 & 5 & 6 \\
\hline 4 & 1 & 1 & & & & \\
5 & 2 & 4 & & & & \\
6 & 6 & 18 & 3 & & & \\
7 & 10 & 45 & 16 & & & \\
8 & 19 & 107 & 72 & 6 & & \\
9 & 28 & 206 & 210 & 39 & & \\
10 & 44 & 381 & 543 & 190 & 10 & \\
11 & 60 & 634 & 1190 & 633 & 76 & \\
12 & 85 & 1025 & 2425 & 1817 & 406 & 15 \\
13 & 110 & 1556 & 4528 & 4480 & 1522 & 130 \\
\hline
\end{tabular}

Table 2 The positive numbers of bipartite non-complete games $B N C G(n, r)$, up to isomorphisms, for $n<14$.

\begin{tabular}{cccc}
\hline$n$ & $B C G(n)$ & $B N C G(n)$ & $B S G(n)$ \\
\hline 1 & 0 & 0 & 0 \\
2 & 1 & 0 & 1 \\
3 & 5 & 0 & 5 \\
4 & 15 & 2 & 17 \\
5 & 36 & 6 & 42 \\
6 & 76 & 27 & 103 \\
7 & 148 & 71 & 219 \\
8 & 273 & 204 & 477 \\
9 & 485 & 483 & 968 \\
10 & 839 & 1168 & 2007 \\
11 & 1424 & 2593 & 4017 \\
12 & 2384 & 5773 & 8157 \\
13 & 3952 & 12326 & 16278 \\
\hline
\end{tabular}

Table 3 The numbers of bipartite non-complete $B N C G(n)$, bipartite complete $B C G(n)$, and bipartite simple games $B S G(n)$, up to isomorphisms, for $n<14$.

3.1 Related work on bipartite simple games for $r=1$ and $r$ being maximal

We summarize here the closed-form expressions of bipartite games that will be useful to generate enumerations of tripartite and quadripartite games from Section 4 to Section 9. We consider the extreme cases for $r$, either minimal $(r=1)$ or maximal.

Formulas for $r=1$ :

Proposition 2 The number of bipartite complete games with one representative of minimal winning coalitions is:

$$
B C G(n, r=1)=(n-1)^{2}
$$

Proof. As the game is bipartite and complete (we assume w.l.o.g. that $N_{1}>N_{2}$ ), $r=1$ implies that the game has vetoes or nulls. Otherwise, if $(a, b)$ was the unique representative of minimal winning coalitions it would be: $a<n_{1}$ ( $a=n_{1}$ means that the $n_{1}$ voters in $N_{1}$ are vetoes $)$ and $b>0\left(b=0\right.$ means that the $n_{2}$ voters in $N_{2}$ are nulls). Then, $(a+1, b-1)$ would be another representative of minimal winning 
coalitions. As neither $(a, b) \geq(a+1, b-1)$ nor $(a+1, b-1) \geq(a, b)$ we have a contradiction with $r=1$.

For each $\left(n_{1}, n_{2}\right)$ with $n_{1}+n_{2}=n\left(n_{i} \geq 1\right.$, for $\left.i=1,2\right)$ there are $n-1$ games with a unique representative of minimal winning coalitions:

- 1 game with vetoes and nulls, which is $\left(n_{1}, 0\right)$,

- $n_{2}-1$ games with vetoes but not nulls, which are $\left(n_{1}, b\right)$ with $1 \leq b \leq n_{2}-1$,

- $n_{1}-1$ games with nulls but not vetoes, which are $(a, 0)$ with $1 \leq a \leq n_{1}-1$.

Hence, each decomposition $\left(n_{1}, n_{2}\right)$ allows for $n-1$ games with $r=1$. As the number of decompositions $\left(n_{1}, n_{2}\right)$ is $n-1$, we conclude that there exist $(n-1)^{2}$ bipartite games with $r=1$.

Proposition 3 The number of bipartite non-complete games with one representative of minimal winning coalitions is:

$$
\operatorname{BNCG}(n, r=1)= \begin{cases}\frac{(n-2)\left(n^{2}-4 n+6\right)}{12}, & \text { if } n \text { is even } \\ \frac{(n-1)(n-2)(n-3)}{12}, & \text { if } n \text { is odd }\end{cases}
$$

Proof. Let $r=1$ and $n=n_{1}+n_{2}$. We claim that:

a. there are $\left(n_{1}-1\right)\left(n_{2}-1\right)$ bipartite non-complete games with $r=1$ for each vector decomposition $\left(n_{1}, n_{2}\right)$ such that $n_{1}>n_{2}$,

b. there are $n(n-2) / 8$ bipartite non-complete games with $r=1$ for each vector decomposition $\left(n_{1}, n_{2}\right)$ such that $n_{1}=n_{2}$.

This is because the game is not complete and whenever $(a, b)$ is the unique representative of minimal winning coalitions it holds that the game has neither vetoes nor nulls (see proof of Proposition 22) so that $0<a<n_{1}$ and $0<b<n_{2}$. The noncompleteness of the game implies that neither $(a+1, b-1)$ nor $(a-1, b+1)$ are winning representatives. From all this we deduce that there $\left(n_{1}-1\right)\left(n_{2}-1\right)$ bipartite non-complete games with $r=1$ for each vector decomposition $\left(n_{1}, n_{2}\right)$ with $n_{1}>n_{2}$, condition required to avoid duplicities of the game. For the particular case, $n_{1}=n_{2}$ we need to subtract from $\left(n_{1}-1\right)\left(n_{2}-1\right)=(n-2)^{2} / 4$ those models $(a, b)$ with $a<b$ (to avoid duplicities). Thus, we obtain $n(n-2) / 8$ when $n$ is even and $n_{1}=n_{2}=n / 2$.

By summing up the number of games for each feasible decomposition $\left(n_{1}, n_{2}\right)$ for $n$, we obtain the expression in $B N C G(n, r=1)$.

It is very interesting to note that the sequence $\operatorname{BNCG}(n, r=1)$ is described in www.oeis.org as A005993, which was also was obtained when enumerating some other problems of different motivation than ours. Observe, therefore, that some enumerating apparently different problems are equivalent to the enumeration of $B N C G(n, r=1)$. 
Corollary 3 The number of bipartite simple games with one representative of minimal winning coalitions is:

$$
B S G(n, r=1)=\left\{\begin{array}{l}
\frac{(n-2)\left(n^{2}-4 n+6\right)}{12}+(n-1)^{2}, \quad \text { if } n \text { is even } \\
\frac{(n-1)(n-2)(n-3)}{12}+(n-1)^{2}, \text { if } n \text { is odd }
\end{array}\right.
$$

The next three results for $r$ being maximal for bipartite complete games are proved in [15], so we only state them here.

Lemma 2 For $n>1, B C G(n, r)=0$ if $r>\left\lceil\frac{n}{2}\right\rceil$.

Proposition 4 Let $n>1$. The number of bipartite complete games with a maximal number of minimal winning pairs $\left\lceil\frac{n}{2}\right\rceil$, is:

$$
\operatorname{BCG}\left(n, r=\left\lceil\frac{n}{2}\right\rceil\right)= \begin{cases}\frac{1}{8}\left(n^{2}+14 n-24\right), & \text { if } n \text { is even } \\ \frac{1}{2}(n-1), & \text { if } n \text { is odd }\end{cases}
$$

Theorem 1 The number of bipartite non-complete simple games, for $n \geq 4$, with a maximal number of minimal winning representatives $\left\lfloor\frac{n}{2}\right\rfloor$, is:

$$
\operatorname{BNCG}\left(n, r=\left\lfloor\frac{n}{2}\right\rfloor\right)= \begin{cases}\frac{1}{8} n(n-2), & \text { if } n \text { is even } \\ \frac{1}{16}\left(n^{3}+n^{2}-25 n+39\right), & \text { if } n \text { is odd }\end{cases}
$$

Note that the polynomials for $n$ even have degree 2 , while the polynomials for $n$ odd have degree 3 . This different behavior for $n$ even and $n$ odd also occurs in this paper when we deal with the case of a maximal number of minimal winning representatives, $r$. The intuition follows from the fact that $r$ is closer to $n$ for $n$ even, than for $n$ odd. This more demanding condition when $n$ is even for $r$ maximal limits the number of games with respect to the odd case for $n$. Observe, for example, that $\operatorname{BNCG}(4,2)=1$ and this game is $\bar{n}=(2,2)$ and $(2,0),(0,2)$ as representatives of minimal winning coalitions, i.e., the bipartite non-complete games defined as $W^{m}=$ $\{\{1,2\},\{3,4\}\} . B N C G(5,2)=4$ and these games are represented by $\bar{n}=(3,2)$ and the respective representatives of minimal winning coalitions:
a. $(3,0),(1,2)$
b. $(3,0),(1,1)$,
c. $(3,0),(0,2)$,
d. $(2,0),(0,2)$.

which respectively correspond to the bipartite non-complete games: 
a. $W^{m}=\{\{1,2,3\},\{1,4,5\},\{2,4,5\},\{3,4,5\}\}$,

b. $W^{m}=\{\{1,2,3\},\{1,4\},\{1,5\},\{2,4\},\{2,5\},\{3,4\},\{3,5\}\}$,

c. $W^{m}=\{\{1,2,3\},\{4,5\}\}$,

d. $W^{m}=\{\{1,2\},\{1,3\},\{2,3\},\{4,5\}\}$.

Corollary 4, deduced from Proposition 4 and Theorem 1 , provides the number of bipartite simple games as a function of $n$ for the maximal number of minimal winning representatives.

\section{Corollary 4}

$$
B S G\left(n, r=\left\lceil\frac{n}{2}\right\rceil\right)= \begin{cases}\frac{1}{4}\left(n^{2}+6 n-12\right), & \text { if } n \text { even } \\ \frac{1}{2}(n-1), & \text { if } n \text { odd }\end{cases}
$$

\section{Enumerations of tripartite simple games with vetoes or nulls}

As pointed out, in Corollary 1. $\operatorname{TNCGV}(n, r)=\operatorname{TNCGN}(n, r)$. Thus, in the rest of this section we only refer to one of them, $T N C G V(n, r)$.

Lemma 3 Let $n$ be the number of players and $r$ be the number of representatives of minimal winning coalitions, then we have

$$
\operatorname{TNCGV}(n, r)=\sum_{i=4}^{n-1} B N C G(i, r)
$$

PROOF. If a bipartite game has either vetoes or nulls then it is a complete game. Thus, all bipartite non-complete games have neither vetoes nor nulls.

From each non-complete bipartite game with $m$ players $(m<n)$ we can add $n-m$ vetoes (or nulls) to get a tripartite non-complete game with $n$ players and vetoes (or nulls) and this is how all non-complete tripartite games with vetoes are generated. Hence, $\operatorname{TNCGV}(n, r)=\sum_{i=4}^{n-1} B N C G(i, r)$.

From Lemma 1 and Lemma 3 we can determine the pairs $(n, r)$, which lead to $\operatorname{TNCGV}(n, r)$ being different of zero.

Corollary $5 \operatorname{TNCGV}(n, r) \neq 0$ if and only if $n>4$ and $r \leq\left\lfloor\frac{n-1}{2}\right\rfloor$.

The number of tripartite non-complete games with vetoes as a function of $n$ can be written more precisely by only using those $B N C G(n, r)$ which are not equal to zero. 


\section{Corollary 6}

$$
T N C G V(n)= \begin{cases}0 & \text { if } n \leq 4 \\ \sum_{i=4}^{n-1} \sum_{r=1}^{\lfloor i / 2\rfloor} B N C G(i, r) & \text { if } n>4\end{cases}
$$

Thus, from Corollary 6 and Table 2 we deduce, in Table 4 , the number of tripartite non-complete games for all feasible combinations $(n, r)$ whenever $n<15$.

\begin{tabular}{crrrrrr}
\hline $\mathrm{n} \downarrow / \mathrm{r} \rightarrow$ & 1 & 2 & 3 & 4 & 5 & 6 \\
\hline 5 & 1 & 1 & & & & \\
6 & 3 & 5 & & & & \\
7 & 9 & 23 & 3 & & & \\
8 & 19 & 68 & 19 & & & \\
9 & 38 & 175 & 91 & 6 & & \\
10 & 66 & 381 & 301 & 45 & & \\
11 & 110 & 762 & 844 & 235 & 10 & \\
12 & 170 & 1396 & 2034 & 868 & 86 & \\
13 & 255 & 2421 & 4459 & 2685 & 492 & 15 \\
14 & 365 & 3977 & 8987 & 7165 & 2014 & 145 \\
\hline
\end{tabular}

Table 4 The positive numbers of tripartite non-complete games with vetoes (or nulls) $T N C G V(n, r)$, up to isomorphisms, for $n<15$.

Table 4 together with Corollary 6 allows to determine the number of tripartite simple games with vetoes (or with nulls) $T S G V$ for small values of $n$. See Table 5 .

\begin{tabular}{cccc}
\hline$n$ & $T C G V(n)$ & $T N C G V(n)$ & $T S G V(n)$ \\
\hline 1 & 0 & 0 & 0 \\
2 & 0 & 0 & 0 \\
3 & 0 & 0 & 0 \\
4 & 1 & 0 & 1 \\
5 & 11 & 2 & 13 \\
6 & 37 & 8 & 45 \\
7 & 98 & 35 & 133 \\
8 & 225 & 106 & 331 \\
9 & 470 & 310 & 780 \\
10 & 919 & 793 & 1712 \\
11 & 1713 & 1961 & 3674 \\
12 & 3082 & 4554 & 7636 \\
13 & 5400 & 10327 & 15727 \\
14 & 9274 & 22653 & 31927 \\
\hline
\end{tabular}

Table 5 The numbers of tripartite games with veto (or null) players: complete $\operatorname{TCGV}(n)$, tripartite noncomplete $T N C G V(n)$, and tripartite simple games $T S G V(n)$, up to isomorphisms, for $n<15$. 


\section{Enumeration of tripartite simple games with either nulls or vetoes with a unique representative of minimal winning coalitions}

The goal of this section is to enumerate the number of tripartite simple games with either nulls or vetoes with a unique representative of minimal winning coalitions. The procedure to follow in this and the subsequent sections is, firstly, determine the number of complete games of this type and, secondly, determine the number of noncomplete games of this type. By the addition of the two results we will obtain the enumeration of tripartite simple games with either vetoes (or nulls) with a unique representative of minimal winning coalitions. This is the first goal of the paper, and as far as we know, a new enumeration.

Proposition 5 The number of tripartite complete games with vetoes (or nulls) with one representative of minimal winning coalitions is:

$$
\operatorname{TCGV}(n, r=1)=\frac{1}{6}\left(n^{3}-6 n^{2}+11 n-6\right) \quad \text { if } n \geq 3
$$

Proof. As the game is complete we have $N_{1}>N_{2}>N_{3}$ and $n_{1}+n_{2}+n_{3}=n$ with $n_{i}>0$ for each $i=1,2,3$. Let $\left(m_{1}, m_{2}, m_{3}\right)$ be the unique minimal winning representative. As the game has vetoes it is $m_{1}=n_{1}$. As the voters in the second class are dominated by the desirability relation by the veto players it must be $m_{2}<n_{2}$, otherwise $m_{2}=n_{2}$ and voters in $N_{2}$ would also be veto players, a contradiction with $N_{1}>N_{2}$. As the voters in the second class dominate by the desirability relation the voters in the third class it must be $m_{2}>0$ and $m_{3}<n_{3}$, otherwise if $m_{2}=0$ or $m_{3}=n_{3}$ the voters in $N_{3}$ would be equally desirable to those in $N_{2}$, a contradiction with $N_{2}>N_{3}$. Then, $n_{1} \geq 1, n_{2} \geq 2$, and $n_{3} \geq 1$.

Hence, given $n$ we need to count all these complete games. For $\left(n_{1}, n_{2}, n_{3}\right)$, with $n_{1} \geq 1, n_{2} \geq 2$, and $n_{3} \geq 1$, we have $n_{2}-1$ choices for $m_{2}$ and $n-n_{2}-1$ choices for the pairs $\left(n_{1}, n_{3}\right)$. By using the subindex $i$ instead of $n_{2}-1$ we can write:

$$
\operatorname{TCGV}(n, r=1)=\sum_{i=1}^{n-3} i \cdot(n-i-2),
$$

which, by induction is equivalent to

$$
\operatorname{TCGV}(n, r=1)=\frac{1}{6}\left(n^{3}-6 n^{2}+11 n-6\right)
$$

Proposition 6 The number of tripartite non-complete games with one representative of minimal winning coalitions is:

$$
T N C G V(n, r=1)=\left\{\begin{array}{l}
\frac{1}{48}\left(n^{4}-10 n^{3}+38 n^{2}-68 n+48\right), \text { if } n \text { is even } \\
\frac{1}{48}\left(n^{4}-10 n^{3}+38 n^{2}-62 n+33\right), \text { if } n \text { is odd }
\end{array}\right.
$$

for $n \geq 5$ and $\operatorname{TNCGV}(n, r=1)=0$ otherwise. 
Proof. By Lemma 6 we know the numbers $\operatorname{TNCGV}(n, r=1)$. We have:

$$
\operatorname{TNCGV}(n, r=1)=\sum_{i=4}^{n-1} B N C G(i, r=1)
$$

for $n \geq 5$ and $\operatorname{TNCGV}(n, r=1)=0$ otherwise. To use the result on bipartite noncomplete games in Proposition 3 we need to distinguish between the even and the odd case. Let $n$ be even. We have:

$$
\begin{aligned}
& \operatorname{TNCGV}(n, r=1)=\sum_{i=1}^{\frac{n}{2}-2} B N C G(n-2 i, r=1)+B N C G(n-(2 i-1), r=1) \\
& \operatorname{TNCGV}(n, r=1)=\frac{1}{12} \sum_{i=1}^{\frac{n}{2}-2}[(n-2 i)(n-2 i-1)(n-2 i-2)+ \\
& \left.(n-2 i-2)\left((n-2 i)^{2}-4(n-2 i)+6\right)\right] \\
& =\frac{1}{48}\left(n^{4}-10 n^{3}+38 n^{2}-68 n+48\right)
\end{aligned}
$$

Let $n$ be odd. We have

$$
\begin{aligned}
\operatorname{TNCGV}(n, r=1) & =\sum_{i=1}^{(n-3) / 2} B N C G(n-(2 i-1), r=1)+\sum_{i=1}^{(n-5) / 2} B N C G(n-2 i, r=1) \\
& =1+\sum_{i=1}^{(n-5) / 2}[B N C G(n-(2 i-1), r=1)+B N C G(n-2 i, r=1)]
\end{aligned}
$$

and by applying the result in Proposition 3 it results:

$$
\begin{aligned}
\operatorname{TNCGV}(n, r=1) & =1+\frac{1}{12} \sum_{i=1}^{(n-5) / 2}\left[( n - 2 i - 1 ) \left((n-2 i+1)^{2}-\right.\right. \\
& 4(n-2 i+1)+6)((n-2 i-1)(n-2 i-2)(n-2 i-3)] \\
& =\frac{1}{48}\left(n^{4}-10 n^{3}+38 n^{2}-62 n+33\right) .
\end{aligned}
$$

The addition of the two results obtained in Propositions 5 and 6 allows to enumerate the class of tripartite simple games with vetoes (or nulls) with a unique minimal winning representative.

Corollary 7 The number of tripartite simple games with vetoes with one representative of minimal winning coalitions is:

$$
\operatorname{TSGV}(n, r=1)= \begin{cases}\frac{1}{48} n \cdot\left(n^{3}-2 n^{2}-10 n+20\right), & \text { if } n \text { is even } \\ \frac{1}{48}\left(n^{4}-2 n^{3}-10 n^{2}+26 n-15\right), & \text { if } n \text { is odd }\end{cases}
$$

Observe that the two polynomials of degree 4 only differ in the linear part. Note that the game described in Example 1 is one of the 5346 tripartite non-complete games with vetoes that are counted in $\operatorname{TNCGV}(n=25, r=1)$ and one of the 7370 tripartite simple games with vetoes that there are counted in $\operatorname{TSGV}(n=25, r=1)$.

The enumerations obtained in Propositions 5 and 6 , and Corollary 7 are, as far as we know, new and candidates to be entered in www.oeis.org. 


\section{Enumeration of tripartite simple games with either vetoes or nulls with a maximal number of minimal winning representatives}

The goal of this section is to enumerate the number of tripartite simple games with either vetoes or nulls with a maximal number of representatives of minimal winning coalitions. We, firstly, determine the number of complete games of this type and, secondly, determine the number of non-complete games of this type. By the addition of the two results we will obtain the enumeration of tripartite simple games with either vetoes (or nulls) with a maximal number of representatives of minimal winning coalitions. This is the second goal of the paper, and as far as we know, a new enumeration.

We start with the complete simple game case. As for $r>1$ there are not bipartite complete games with vetoes, we have:

$$
T C G V(n, r)=\sum_{i<n} B C G(i, r)
$$

whenever $r>1$. The maximal value for $r$ as a function of $n$ is achieved for $n=\left\lfloor\frac{n}{2}\right\rfloor$ because of Lemma 2 and that

$$
\operatorname{TCGV}\left(n, r=\left\lfloor\frac{n}{2}\right\rfloor\right)= \begin{cases}B C G\left(n-1,\left\lfloor\frac{n}{2}\right\rfloor\right) & \text { if } n \text { is even } \\ B C G\left(n-1,\left\lfloor\frac{n}{2}\right\rfloor\right)+B C G\left(n-2,\left\lfloor\frac{n}{2}\right\rfloor\right) & \text { if } n \text { is odd }\end{cases}
$$

From this equality and Proposition 4 we deduce the next result.

Proposition 7 For $n \geq 4$ :

$$
\operatorname{TCGV}\left(n, r=\left\lfloor\frac{n}{2}\right\rfloor\right)= \begin{cases}\frac{1}{2}(n-2), & \text { if } n \text { is even } \\ \frac{1}{8}\left(n^{2}+16 n-49\right), & \text { if } n \text { is odd }\end{cases}
$$

We consider now non-complete games.

Proposition 8 For $n \geq 5$ :

$$
\operatorname{TNCGV}\left(n, r=\left\lfloor\frac{n-1}{2}\right\rfloor\right)= \begin{cases}\frac{1}{16}\left(n^{3}-36 n+80\right), & \text { if } n \text { is even } \\ \frac{1}{8}(n-1)(n-3), & \text { if } n \text { is odd }\end{cases}
$$

PROOF. We apply Theorem 1 and Corollary 6 on bipartite non-complete games and we distinguish between the odd and the even case.

Let $n$ be an even number, then

$$
\begin{aligned}
\operatorname{TNCGV}\left(n, r=\left\lfloor\frac{n-1}{2}\right\rfloor\right) & =B N C G(n-1,(n-2) / 2)+B N C G(n-2,(n-2) / 2) \\
& =\frac{1}{16}\left((n-1)^{3}+(n-1)^{2}-25(n-1)+39\right)+\frac{1}{8}(n-2)(n-4) \\
& =\frac{1}{16}\left(n^{3}-36 n+80\right) .
\end{aligned}
$$


Let $n$ be an odd number, then

$$
\operatorname{TNCGV}\left(n, r=\left\lfloor\frac{n-1}{2}\right\rfloor\right)=\operatorname{BNCG}(n-1,(n-1) / 2)=\frac{1}{8}(n-1)(n-3) .
$$

Now the polynomials for $n$ even have degree 3 and the polynomials for $n$ odd have degree 2 (oppositely to what occurs for $B N C G(n, r=\lfloor n / 2\rfloor)$ ). But observe that for consecutive numbers of $n, r$ is closer to $n$ for $n$ odd than for $n$ even. Thus, the number of non-complete tripartite games with vetoes increases faster for $n$ even than for $n$ odd.

By using the results obtained in Propositions 7 and 8 we deduce the sequence for the number of simple games with a maximal number of minimal winning representatives.

Corollary 8 For $n \geq 4$ :

$$
\operatorname{TSGV}\left(n, r=\left\lfloor\frac{n}{2}\right\rfloor\right)= \begin{cases}\frac{1}{2}(n-2), & \text { if } n \text { is even } \\ \frac{1}{4}\left(n^{2}+6 n-23\right), & \text { if } n \text { is odd }\end{cases}
$$

The enumerations obtained in Propositions 7 and 8 and in Corollary 8 are, as far as we know, new candidates to be entered in www.oeis.org.

\section{Enumeration of quadripartite simple games with vetoes and nulls}

The next Lemma 4 links non-complete quadripartite simple games with non-complete bipartite simple games so that we can easily enumerate these games for small combinations of $n$ and $r$.

Lemma 4 Given $n>5$ and $r \leq\left\lfloor\frac{n}{2}\right\rfloor-1$,

$$
Q N C G V N(n, r)=\sum_{i=1}^{n-(2 r+1)} i \cdot B N C G(n-i-1, r)
$$

PROOF. A bipartite non-complete game does not have vetoes or nulls. On the other hand, any quadripartite non-complete game with vetoes and nulls of $n$ players and $r$ representatives of minimal winning coalitions can be obtained from a bipartite noncomplete game of $r$ representatives and with $j$ voters, with $j<n-1$. It is only needed to add $k$ vetoes and $j-k$ nulls to the bipartite game, where $0<k<j$. Thus, 
$n-j-1$ games of this type are obtained from bipartite games with $j$ voters. Let $j=n-i-1$, then it holds

$$
\operatorname{QNCGVN}(n, r)=\sum_{i=1}^{n-(2 r+1)} i \cdot B N C G(n-i-1, r)
$$

From Lemma 4 and the enumeration of non-complete bipartite games in Table 2 we can determine the number of quadripartite non-complete games with vetoes and nulls in Table 6

\begin{tabular}{crrrrrr}
\hline $\mathrm{n} \downarrow / \mathrm{r} \rightarrow$ & 1 & 2 & 3 & 4 & 5 & 6 \\
\hline 6 & 1 & 1 & & & & \\
7 & 4 & 6 & & & & \\
8 & 13 & 29 & 3 & & & \\
9 & 32 & 97 & 22 & & & \\
10 & 70 & 272 & 113 & 6 & & \\
11 & 136 & 653 & 414 & 51 & & \\
12 & 246 & 1415 & 1258 & 286 & 10 & \\
13 & 416 & 2811 & 3292 & 1154 & 96 & \\
14 & 671 & 5242 & 5232 & 3839 & 588 & 15 \\
15 & 1036 & 9249 & 16738 & 11004 & 2602 & 160 \\
\hline
\end{tabular}

Table 6 The positive numbers of quadripartite non-complete games with veto players and nulls $Q N C G V N(n, r)$, up to isomorphisms, for $n<16$.

The numbers in Table 6 together with the enumeration for quadripartite complete games with vetoes and nulls, in Equation (3), leads to the enumeration of the number of quadripartite simple games with vetoes and nulls $Q S G V N$ for small values of $n$ in Table 7

\section{Enumeration of quadripartite simple games with vetoes and nulls with a unique representative of minimal winning coalitions}

The goal of this section is to enumerate the number of quadripartite simple games with vetoes and nulls with a unique representative of minimal winning coalitions.

Proposition 9 There are no quadripartite complete games with vetoes and nulls with a unique minimal winning representative.

Proof. Assume there exists a complete game of this type. Then, by completeness it is $N_{1}>N_{2}>N_{3}>N_{4}$. Let $\left(m_{1}, m_{2}, m_{3}, m_{4}\right)$ be the unique minimal winning representative. It must verify:

a. $m_{1}=n_{1}$, because the strongest players by the desirability relation are vetoes. 


\begin{tabular}{cccc}
\hline$n$ & $Q C G V N(n)$ & $Q N C G V N(n)$ & $Q S G V N(n)$ \\
\hline 1 & 0 & 0 & 0 \\
2 & 0 & 0 & 0 \\
3 & 0 & 0 & 0 \\
4 & 0 & 0 & 0 \\
5 & 0 & 0 & 0 \\
6 & 8 & 2 & 10 \\
7 & 35 & 10 & 45 \\
8 & 113 & 45 & 158 \\
9 & 303 & 151 & 454 \\
10 & 717 & 461 & 1178 \\
11 & 1552 & 1254 & 2806 \\
12 & 3145 & 3215 & 6360 \\
13 & 6062 & 7769 & 13831 \\
14 & 11242 & 15587 & 26829 \\
15 & 20230 & 40789 & 61019 \\
\hline
\end{tabular}

Table 7 The numbers of quadripartite games with vetoes and nulls: complete games $\operatorname{QCGVN}(n)$, noncomplete games $\operatorname{QNCGVN}(n)$, and simple games $\operatorname{QSGVN}(n)$, up to isomorphisms, for $n<16$.

b. $m_{2}<n_{2}$, otherwise the players in $N_{2}$ would also be vetoes, a contradiction with $N_{1}>N_{2}$.

c. $m_{2}>0$, otherwise the players in $N_{2}$ and in $N_{3}$ would belong to the same equivalence class, a contradiction with $N_{2}>N_{3}$.

d. $m_{3}<n_{3}$, otherwise the players in $N_{3}$ and in $N_{2}$ would belong to the same equivalence class, a contradiction with $N_{2}>N_{3}$.

e. $m_{3}>0$ otherwise the players in $N_{3}$ would be nulls too, a contradiction.

f. $m_{4}=0$, because these players are nulls.

But, then $\left(n_{1}, m_{2}+1, m_{3}-1,0\right)$ would also be a minimal winning representative. Thus, a contradiction with the assumed condition $r=1$.

As a consequence of Proposition 9 we have $Q N C G V N(n, r=1)=Q S G V N(n, r=$ $1)$. We now compute these numbers.

Proposition 10 The number of quadripartite non-complete games with vetoes and nulls with one representative of minimal winning coalitions is:

$\operatorname{QNCGVN}(n, r=1)=\left\{\begin{array}{l}\frac{1}{240}\left(n^{5}-15 n^{4}+90 n^{3}-270 n^{2}+404 n-240\right), \text { if } n \text { is even } \\ \frac{1}{240}\left(n^{5}-15 n^{4}+90 n^{3}-270 n^{2}+389 n-195\right), \text { if } n \text { is odd }\end{array}\right.$

Proof. By using Lemma 4 for $r=1$ we have

$$
\operatorname{QNCGVN}(n, r=1)=\sum_{i=1}^{n-5} i \cdot \operatorname{BNCG}(n-i-1, r=1)
$$

We now replace $B N C G(n-i-1, r=1)$ for its value by distinguishing the even from the odd case. 
Let $n$ be even. We have:

$$
\begin{aligned}
\operatorname{QNCGVN}(n, r=1)= & \frac{1}{12} \sum_{i=1}^{n / 2-2}(2 i-1)(n-2 i-2)\left((n-2 i)^{2}-4(n-2 i)+6\right)+ \\
& \frac{1}{12} \sum_{i=1}^{n / 2-3}(2 i)(n-2 i-2)((n-2 i-3)(n-2 i-4) .
\end{aligned}
$$

Let $n$ be odd. We have

$$
\begin{aligned}
\operatorname{QNCGVN}(n, r=1)= & \frac{1}{12} \sum_{i=1}^{(n-1) / 2-2}(2 i-1)(n-2 i-1)(n-2 i-2)(n-2 i-3)+ \\
& \frac{1}{12} \sum_{i=1}^{(n-1) / 2-2}(2 i)(n-2 i-3)\left((n-2 i-1)^{2}-4(n-2 i-1)+6\right) .
\end{aligned}
$$

Expressions that after simplification become

$\operatorname{QNCGVN}(n, r=1)=\left\{\begin{array}{l}\frac{1}{240}\left(n^{5}-15 n^{4}+90 n^{3}-270 n^{2}+404 n-240\right), \text { if } n \text { is even } \\ \frac{1}{240}\left(n^{5}-15 n^{4}+90 n^{3}-270 n^{2}+389 n-195\right), \text { if } n \text { is odd }\end{array}\right.$

Note that these two polynomials of degree 5 just differ in the linear part.

As there are no quadripartite complete games with vetoes and nulls for $r=1$, according to Proposition 9, the next consequence trivially follows.

Corollary 9 The number of quadripartite simple games with vetoes and nulls with one representative of minimal winning coalitions is:

$Q S G V N(n, r=1)=\left\{\begin{array}{l}\frac{1}{240}\left(n^{5}-15 n^{4}+90 n^{3}-270 n^{2}+404 n-240\right), \text { if } n \text { is even } \\ \frac{1}{240}\left(n^{5}-15 n^{4}+90 n^{3}-270 n^{2}+389 n-195\right), \text { if } n \text { is odd }\end{array}\right.$

The enumeration obtained in Corollary 9 is, as far as we know, a new and a candidate to be entered in www.oeis.org.

\section{Enumeration of quadripartite simple games with vetoes and nulls with a maximal number of minimal winning representatives}

We start by computing the number of complete games of this type. The maximal value for $r$ as a function of $n$ when computing $\operatorname{QCGVN}(n, r)$ is achieved for $r=\left\lfloor\frac{n-1}{2}\right\rfloor$ 
because of Lemma 2 and that it is necessary to add at least a veto player and a null player in bipartite complete games with a maximal $r$, which implies that

$\operatorname{QCGVN}\left(n, r=\left\lfloor\frac{n-1}{2}\right\rfloor\right)= \begin{cases}B C G\left(n-2,\left\lfloor\frac{n-1}{2}\right\rfloor\right)+2 \cdot B C G\left(n-3,\left\lfloor\frac{n-1}{2}\right\rfloor\right) & \text { if } n \text { is even } \\ B C G\left(n-2,\left\lfloor\frac{n-1}{2}\right\rfloor\right) & \text { if } n \text { is odd }\end{cases}$

From this equality and Proposition 4 we deduce the next result.

Proposition 11 For $n \geq 5$ :

$$
Q C G V N\left(n, r=\left\lfloor\frac{n-1}{2}\right\rfloor\right)= \begin{cases}\frac{1}{8}\left(n^{2}+18 n-80\right), & \text { if } n \text { is even } \\ \frac{1}{2}(n-3), & \text { if } n \text { is odd }\end{cases}
$$

Proposition 12 The number of quadripartite non-complete games with vetoes and nulls with a maximal number of minimal winning representatives is:

QNCGVN $(n, r=\lfloor n / 2\rfloor-1)= \begin{cases}\frac{1}{8}(n-2)(n-4), & \text { if } n \text { is even } \\ \frac{1}{16}\left(n^{3}-n^{2}-49 n+145\right), & \text { if } n \geq 5 \text { is odd }\end{cases}$

PROOF. Let $n$ be an even number. We have:

$\operatorname{QNCGVN}(n, r=n / 2-1)=\operatorname{BNCG}(n-2, r=(n-2) / 2)=\frac{1}{8}(n-2)(n-4)$.

Let $n$ be an odd number. We have:

$$
\begin{aligned}
\operatorname{QNCGVN}\left(n, r=\frac{n-3}{2}\right) & =2 \cdot \operatorname{BNCG}\left(n-3, \frac{n-3}{2}\right)+1 \cdot \operatorname{BNCG}\left(n-2, \frac{n-3}{2}\right) \\
& =\frac{2}{8}(n-3)(n-5)+\frac{1}{16}\left((n-2)^{3}+(n-2)^{2}-25(n-2)+39\right) \\
& =\frac{1}{16}\left(n^{3}-n^{2}-49 n+145\right) .
\end{aligned}
$$

Note again that for consecutive values of $n, r$ is closer to $n$ for $n$ even than for $n$ odd, which allows a faster increasing of the number of games for $n$ odd.

By adding the results in Proposition 11 and Proposition 12 we obtain, in Corollary 10 , the number of quadripartite simple games with vetoes and nulls.

Corollary 10 For $n \geq 5$ :

$$
\operatorname{QSGVN}\left(n, r=\left\lfloor\frac{n-1}{2}\right\rfloor\right)= \begin{cases}\frac{1}{4}\left(n^{2}+6 n-36\right), & \text { if } n \text { is even } \\ \frac{1}{2}(n-3), & \text { if } n \text { is odd }\end{cases}
$$

The enumerations obtained in Proposition 12 and Corollary 10 are, as far as we know, new and candidates to be entered in www.oeis.org. 


\section{Conclusion and open problems}

This article finds concise closed-form expressions for certain classes of monotonic Boolean functions or, equivalently, certain classes of simple games. Specifically, games are enumerated, in which the players are grouped into three or four equivalence classes and contain either very powerful voters (with the right to veto) or weak voters without the ability to influence (nulls). The results that are obtained are polynomial.

From a theoretical point of view, the results obtained contribute to enlarging and expanding the known enumerations of some subclasses of Boolean functions, which constitute a variant of Dedekind's problem on the enumeration of monotonic inequivalent Boolean functions or simple games.

From a practical point of view, the results obtained contribute to the design of suitable voting systems that meet certain conditions desired a priori. For instance, if one wants to design a tricameral voting system with veto players and with a given number of representatives, then he/she can generate all of them through the results of this article and select the most convenient one. Similarly, the results can be used to design circuits or reliability systems with certain restrictions.

Listing monotonic Boolean functions is a tremendously complex problem, Dedekind already noticed it. Enumerating subclasses of them is not easy either, for example the number of bipartite simple games is already exponential. We conclude this paper by indicating two open problems that derive from this paper and that are likely to be studied in the near future.

We have determined the number of tripartite and quadripartite simple games with special types of voters. It is an open and challenging problem to determine the number of tripartite simple games and quadripartite simple games. Given its complexity, Soft computing techniques might be very useful to achieve this ambitious goal.

The most relevant problem in Boolean functions is to determine whether a given function is threshold. As 2-monotonicity is a necessary, but not sufficient condition for the function to be threshold, it is of great interest to enumerate classes of 2monotonic functions and then check one-by-one which of them are threshold, since this checking can be done in polynomial time. A direct continuation of this paper would be to enumerate the number of weighted tripartite simple games with vetoes (or nulls) and the number of quadripartite simple games with vetoes and nulls.

\section{Acknowledgments}

I'm very grateful to three anonymous referees whose interesting comments allowed me to improve the paper. 


\section{Compliance with Ethical Standards}

Funding: This study was partially funded by funds from the Spanish Ministry of Science and Innovation grant PID2019-I04987GB-I00

Conflict of Interest: I declare that I has no conflict of interest.

Ethical approval (in case of humans or animals were involved): This article does not contain any studies with human participants or animals performed by any of the authors.

\section{References}

1. Boros E, Hammer PL, Ibaraki T, and Kawakawi K (1991) Identifying 2-monotonic positive Boolean functions in polynomial time, in ISA'91 Algorithms, W.L. Hsu and R.C.T. Lee eds., Springer Lecture Notes in Computer Sciences (LNCS), 557:104-115.

2. Boros E, Hammer PL, Ibaraki T, and Kawakawi K (1997) Polynomial time recognition of 2monotonic positive functions given by an oracle, SIAM Journal of Computing, 26:93-109.

3. Carreras F and Freixas J (1996) Complete simple games, Mathematical Social Sciences, 32:139-155

4. Crama Y (1987) Dualization of regular Boolean functions, Discrete Applied Mathematics, 16:79-85.

5. Chvátal V and Hammer PL (1977) Aggregation of inequalities in integer programming, Annals of Discrete Mathematics, 1:145-162.

6. Cheung WS and Ng TW (2014) A three-dimensional voting system in Hong Kong, European Journal of Operational Research, 236: 292-297.

7. Einy E (1985) The desirability relation of simple games, Mathematical Social Sciences 10:155-168

8. Felsenthal DS and Machover M (1998) The measurement of voting power, Edward Elgar Publishing Limited, Cheltenham, UK

9. Freixas J (2004) The dimension for the European Union Council under the Nice rules, European Journal of Operational Research, 156:415-419.

10. Freixas J, Freixas M, and Kurz S (2017) On the characterization of weighted simple games, Theory and Decision, 83(4): 469-498

11. Freixas J and Kurz S (2013) The golden number and Fibonacci sequences in the design of voting systems, European Journal of Operational Research, 226: 246-257.

12. Freixas J and Molinero X (2009) Simple games and weighted games: a theoretical and computational viewpoint, Discrete Applied Mathematics, 157:1496-1508.

13. Freixas J, Molinero X, and Roura S (2012) Complete voting systems with two types of voters: weightedness and counting, Annals of Operations Research, 193:273-287.

14. Freixas J and Puente MA (2002) Reliability importance measures of the components in a system based on semivalues and probabilistic values, Annals of Operations Research, 109:331-342.

15. Freixas J and Samaniego D (2020) On the enumeration of bipartite simple games, Discrete Applied Mathematics, submitted.

16. Gvozdeva T, Hameed A, and Slinko A (2013) Weightedness and structural characterization of hierarchical simple games, Mathematical Social Sciences, 65:181-189.

17. Hardy GH (1999) Ramanujan: Twelve lectures on subjects suggested by his life and work, 3rd ed. New York: Chelsea.

18. Hu ST (1965) Threshold Logic, Univ. of California Press, Berkeley and Los Angeles, USA.

19. Isbell JR (1958) A class of simple games, Duke Mathematics Journal, 25:423-439.

20. Kilgour M (1983) A formal analysis of the amending formula of Canada's Constitution Act, Canadian Journal of Political Science, 16:771-777.

21. Kuo W and Zhu X (2012) Importance measures is reliability, risk, and optimization: principles and applications, John Wiley \& Sons, West Sussex, United Kingdom.

22. Kurz S (2012) On minimum sum representations for weighted voting games, Annals of Operations Research, 196(1):361-369.

23. Kurz S and Napel S (2016) A dimension of the Lisbon voting rules in the EU Council: a challenge and new world record, Optimization Letters, 10(6):1245-1256 
24. Kurz S and Tautenhahn N (2013) On Dedekind's problem for complete simple games, International Journal Game Theory 42:411-437

25. Le Breton M, Montero M, and Zaporozhets V (2012) Voting power in the EU council of ministers and fair decision making in distributive politics, Mathematical Social Sciences, 63:159-173.

26. Leech D (2002) Voting power in the governance of the International monetary fund, Annals of Operations Research, 109:375-397.

27. Marzi F, Rossi F and Smriglio S (2019) Computational study of separation algorithms for clique inequalities, Soft Computing, 23:3013-3027.

28. Muroga S (1971) Threshold Logic and its Applications, Wiley-Interscience, New York.

29. Peters H (2015) Game theory: A multi-leveled approach, second edition. Springer, the Netherlands.

30. Picton PD (1991) Neural Networks, second edition. Macmillan, Indianapolis.

31. Ramamurthy KG (1990) Coherent Structures and Simple Games, Theory and Decision Series C, Springer, Netherlands.

32. Reiterman J, Rödl V, Siňajová E, and Tuma M, (1985) Threshold hypergraphs, Discrete Mathematics, 54:193-200

33. Roychowdhury V, Siu K, and Orlitsky A (1994) Theoretical Advances in Neural Computation and Learning, Norwell, MA: Kluwer

34. Sloane, NJA (1964) www.oeis.org Encyclopedia On-Line of Integer Sequences

35. Siu K, Roychowdhury V, and Kailath T (1995) Discrete Neural Computation: A Theoretical Foundation, Englewood Cliffs, NJ: Prentice-Hall.

36. Simmons GJ (1990) How to (really) share a secret, In: Proceedings of the 8th Annual International Cryptology Conference on Advances in Cryptology, pages 390-448. Springer-Verlag, London, UK.

37. Stinson DR (1992) An explication of secret sharing schemes, Designs, Codes and Cryptography, 2:357-390.

38. Tassa T (2007) Hierarchical threshold secret sharing, Journal of Cryptology, 20:237-264.

39. Taylor AD and Pacelli A (2008) Mathematics and Politics, second edition. Springer Verlag, New York, USA.

40. Taylor AD and Zwicker WS (1992) A characterization of weighted voting, Proceedings of the American Mathematical Society, 115:1089-1094.

41. Taylor AD and Zwicker WS (1993) Weighted voting, multicameral representation, and power, Games and Economic Behavior, 5:170-181

42. Taylor AD and Zwicker WS (1999) Simple games: desirability relations, trading, and pseudoweightings, Princeton University Press, New Jersey, USA.

43. von Neumann J and Morgenstern O (1944) Theory of games and economic behavior, Princeton University Press, New Jersey, USA. 